\title{
Nueva metodología de valoración y seguimiento de riberas fluviales y técnicas de gestión aplicadas a varios cauces del norte de España
}

\author{
New methodology for the valuation and monitoring of riverbanks \\ and management techniques applied to several riverbeds in northern Spain
}

\author{
Ana Isabel Magide Herrero ${ }^{a *}$, Juan Andrés Oria de Rueda Salgueiro ${ }^{b}$ \\ *Autor de correspondencia: a TALHER S.A, Valladolid, España, tel.: 0034-600936036, aimagide@talher.com \\ ${ }^{\mathrm{b}}$ Universidad de Valladolid, ETSII Agrarias, Palencia, España.
}

\begin{abstract}
SUMMARY
In the present work, a new method for the environmental assessment of riverbanks integrating the Forest Stewardship Council (FSC) certification scheme is proposed. The method collects and evaluates the attributes detailed in the Glossary of Spanish Forest Management Standards for FSC certification, which enabled the detection of many High Conservation Value Forests (MAVC). FSC defines High Conservation Value Forests as those that contain one or more of the biological, social and cultural attributes considered of critical or exceptional importance to a certain area. As the included indicators were objectified, results did not depend on subjectivity and could be compared to different areas studied by different people. At first, this method was applied in small plots in northern Spain and FSC forest certification was obtained. Later it was applied in two rivers, Carrión and Valdavia, located in the northern Spanish sub-plateau. In all cases, application was simple, no values were detected in the areas studied that were not in accordance with the method. Moreover, the use of the FSC certification scheme with international implantation allowed us to consider applying this method in other riverbanks around the world.
\end{abstract}

Key words: riparian forest, priority habitats, conservation, biodiversity, FSC certification.

\section{RESUMEN}

En el presente trabajo se propuso un nuevo método para la valoración ambiental de riberas fluviales, que integró el esquema de certificación Forest Stewardship Council (FSC) o Consejo de Administración Forestal. El método recogía y valoraba los atributos detallados en el Glosario de los Estándares Españoles de Gestión Forestal para la certificación FSC, lo que permitió detectar los Montes de Alto Valor de Conservación (MAVC). Los MAVC eran definidos por FSC como aquellos montes que contienen uno o más de los atributos biológicos, sociales y culturales considerados de importancia crítica o excepcional para cierta zona. Los indicadores incluidos se objetivaron para que los resultados no dependieran de la subjetividad y se pudieran comparar zonas diferentes estudiadas por distintas personas. En un primer momento, este método se aplicó en pequeñas parcelas del norte de España y se obtuvo la certificación forestal FSC. Posteriormente se aplicó en los cauces de dos ríos con características muy diferentes, Carrión y Valdavia, situados en la submeseta norte española. En todos los casos la aplicación fue sencilla, no detectándose valores en las zonas estudiadas que no fuesen recogidos por el método. Además, la utilización del esquema de certificación FSC con implantación internacional permitió considerar la posibilidad de utilizar este método en diferentes riberas del mundo.

Palabras clave: bosque ribereño, hábitats prioritarios, conservación, biodiversidad, certificación FSC.

\section{INTRODUCCIÓN}

La valoración y el seguimiento de las riberas fluviales es fundamental para poder realizar una buena gestión de los ecosistemas, que proteja, conserve y mejore el estado ecológico de las mismas, tal y como exige la Directiva Marco de Aguas (2.000/60/CE) a los estados europeos miembros (González del Tánago et al. 2006). Actualmente existen numerosas metodologías para la valoración ambiental de las riberas, en las que se abordan aspectos relativos a una gran superficie con una elevada longitud de ribera, entre las que cabe destacar las propuestas hechas por Petersen (1992), Munné et al. $(1998,2003)$ con el índice de calidad del bosque de ribera (QBR), Winward (2000), Bjorkland et al. (2001), Pardo et al. (2002) con el índice de Hábitat Fluvial (IHF), Snelder y Biggs (2002) con el River Environment Classification (REC), Ward et al. (2003), Jansen et al. (2004), y González del Tánago et al. (2006) con el Riparian Quality Index (RQI).

Algunos de estos métodos hacen especial hincapié en la estructura de la vegetación, (Munné et al. 1998, 2003, Winward 2000). Otros se basan más en las dimensiones de 
la ribera, la calidad del hábitat y el uso del suelo (Petersen 1992, Bjorkland et al. 2001, Ward et al. 2003, Jansen et al. 2004 y González del Tánago et al. 2006). En el caso del IHF (Pardo et al. 2002), el método tiene en cuenta únicamente el estado del cauce, mientras que el REC (Snelder y Biggs 2002), realizado para ríos neozelandeses, se basa en aspectos climáticos, morfológicos y geológicos. Este último método es adaptado por Peredo-Parada et al. 2011 a los ríos de Chile por presentar muchas similitudes eco-hidrológicas entre estos ríos y los de Nueva Zelanda. Estas similitudes son el régimen hidrológico, geomorfología fluvial, presencia de ciertas especies de peces y numerosas especies de macro invertebrados (Peredo-Parada et al. 2011).

Los métodos anteriores incorporan mayoritariamente criterios naturales, dejando fuera otra serie de criterios que pueden tener su importancia, tales como criterios culturales, sociales y/o espirituales. Además, esos métodos valoran las riberas utilizando a las especies arbóreas como casi único factor de interés de conservación, no considerando otros hábitats que también pueden ser muy valiosos como pastizales, gleras de piedra o juncales, entre otros. Incluso, no consideran la existencia de madera muerta de elevado diámetro que constituye un valioso microhábitat, dando refugio y protección a muchas especies animales (Emberger et al. 2015). Por otra parte, existen métodos sujetos a la subjetividad de la persona que lo aplica y fundamentalmente, no tienen en cuenta ciertos atributos necesarios para que un lugar sea certificado por Forest Stewardship Council (FSC).

Ante las limitaciones observadas en las metodologías anteriores surge este trabajo, cuyo objetivo principal es desarrollar una nueva metodología de valoración y seguimiento de riberas fluviales. Esta metodología incluye un modelo de valoración ambiental novedoso, práctico y sencillo, basado en criterios culturales, sociales y/o espirituales, además de los naturales, y que considera interesantes para la conservación otros hábitats aparte de los vinculados al estrato arbóreo. Además, se plantean otros objetivos como que la nueva metodología recoja por primera vez un conjunto variado de atributos necesarios para conseguir la certificación forestal FSC, de forma que se facilite la venta de la madera existente en estos lugares, así como la recepción de subvenciones. Por otra parte estos atributos marcados por FSC también permiten detectar zonas que puedan ser declaradas Montes de Alto Valor de Conservación (MAVC) por reconocerse una o varias de estas características sobre el terreno estudiado. Los MAVC son definidos por FSC como aquellos montes que contienen uno o más de los atributos biológicos, sociales y culturales considerados de importancia crítica o excepcional para cierta zona. La certificación FSC es un sello de calidad que garantiza que todo producto forestal que lo lleva ha sido obtenido de un bosque desarrollado según los principios y criterios de buena gestión que establece el propio consejo, y que son reconocidos internacionalmente. Este reconocimiento y uso internacional del esquema de certificación FSC hace considerar la posibilidad de aplicar este método en otras partes del mundo. Por último, a base de los resultados de la metodología desarrollada, un tercer objetivo del trabajo consiste en plantear medidas técnicas prácticas y viables de actuación adaptadas a las características de la zona centro-norte de España según se quiera mantener o mejorar el estado de conservación de las zonas objeto de estudio.

\section{MÉTODOS}

Establecimiento de la metodología para determinar el estado de conservación. La nueva metodología propuesta para determinar el estado de conservación de una ribera se basó en la ponderación cualitativa de quince atributos considerados de gran importancia en la conservación. Para esta ponderación se consideraron trabajos de evaluación ambiental y de biodiversidad realizados en otros países Emberger et al. (2015), así como bibliografía especializada que resalta la gran importancia del mosaico de paisajes para la conservación (Dufour y Piégay 2006, Abell et al. 2015). La evaluación global de cada tramo de ribera se calcula como suma de las puntuaciones obtenidas en cada uno de los atributos fundamentales de acuerdo a los criterios de valoración establecidos. Los quince atributos considerados, así como los correspondientes criterios de puntuación, se indican a continuación y se resumen posteriormente en el cuadro 1. Para interpretar el resultado obtenido, se establecieron unas categorías cualitativas según el valor de conservación obtenido de la suma anterior, tal y como queda reflejado en el cuadro 2. Los 15 atributos de valoración son:

1. Número de especies autóctonas de árboles. En los ecosistemas ribereños degradados y simplificados, el número de especies decrece notablemente tras las perturbaciones artificiales fuertes, disminuyendo la diversidad arbórea hasta una o dos especies como máximo. Por el contrario, los parajes de sotos con un mínimo de cinco especies arbóreas indican un estado natural claramente más positivo (Dufour y Piégay 2006). De este modo, la máxima puntuación posible en el presente apartado (5 puntos) se obtendría con un total de cinco especies o más, con tres o cuatro especies se obtendría una puntuación de 2; y con dos especies o menos no se obtendría puntuación.

2. Abundancia de madera muerta de elevado diámetro. Valioso microhábitat con valor indicativo de refugio y protector de fauna. Se incluyeron troncos derribados y ramas, así como tocones, siempre y cuando presentasen un diámetro mayor de $20 \mathrm{~cm}$ (Camprodon 2008). Se otorgaron 2 o 5 puntos en función de si los hábitats presentaban respectivamente escasa (1-5 ejemplares/ ha) o abundante ( $>5$ ejemplares/ha) madera muerta.

3. Presencia de espinares y zarzales. Formados por arbustos espinosos. Valioso hábitat de refugio para la fauna 
terrestre y aves. Se valoró con 5 puntos la existencia de zarzales no espesos o irregulares mientras que si eran espesos y bien delimitados formando ecotonos la puntuación era 10. Cuando estas formaciones no aparecían o eran pies aislados se contabilizó 0 puntos.

4. Densidad árboles vivos gruesos. Estos árboles son un destacado refugio y lugar de cría de aves y mamíferos, por lo que se consideró imprescindible un mínimo de cinco árboles maduros por hectárea (diámetro mayor de $45 \mathrm{~cm}$ ) (Camprodon 2008), como indicador de estado favorable de un ecosistema forestal. De este modo se asignó una puntuación de 0 cuando eran inexistentes, de 2 cuando eran escasos (1-5 pies/ha) y 5 cuando eran numerosos ( $>5 \mathrm{pies} / \mathrm{ha})$.

5. Densidad de Árboles vivos portadores de microhábitats. Se contabilizó el número de árboles que presenta-

Cuadro 1. Ficha de ponderación de variables cualitativas de conservación.

Data sheet weighing of qualitative variables of conservation.

\begin{tabular}{|c|c|}
\hline Atributo & Criterio de puntuación \\
\hline Especies autóctonas de árboles & $\begin{array}{l}0: 0,1 \text { o } 2 \text { especies } \\
\text { 2: } 3 \text { o } 4 \text { especies } \\
\text { 5: } 5 \text { especies o más }\end{array}$ \\
\hline Madera muerta de elevado diámetro & $\begin{array}{l}\text { 0: sin madera muerta } \\
\text { 2: madera muerta escasa (entre } 1 \text { y } 5 \text { ejemplares/ha) } \\
\text { 5: madera muerta frecuente (más de } 5 \text { ejemplares/ha) }\end{array}$ \\
\hline Espinares y zarzales & $\begin{array}{l}\text { 0: no aparecen representados o prácticamente inexistentes } \\
\text { 5: existencia de zarzales no espesos e irregulares } \\
\text { 10: zarzales espesos y bien delimitados como ecotonos }\end{array}$ \\
\hline Árboles de elevado diámetro & $\begin{array}{l}\text { 0: inexistentes } \\
\text { 2: escasos (entre } 1 \text { y } 5 \text { ejemplares/ha) } \\
\text { 5: numerosos (más de } 5 \text { ejemplares/ha) }\end{array}$ \\
\hline Árboles vivos portadores de microhábitats & $\begin{array}{l}\text { 0: inexistentes } \\
\text { 2: escasos (entre } 1 \text { y } 5 \text { ejemplares/ha) } \\
\text { 5: numerosos (más de } 5 \text { ejemplares/ha) }\end{array}$ \\
\hline Medios abiertos con plantas con flores & $\begin{array}{l}0 \text { : inexistentes } \\
\text { 2: escasos (menos de un } 10 \% \text { de superficie) } \\
\text { 5: bien representados (igual o mayor al } 10 \% \text { de superficie) }\end{array}$ \\
\hline Medios acuáticos: humedales y estanques & $\begin{array}{l}0 \text { : inexistentes } \\
\text { 2: escasos (menos de un } 10 \% \text { de superficie) } \\
\text { 5: bien representados (igual o mayor al } 10 \% \text { de superficie) }\end{array}$ \\
\hline Medios pedregosos, gleras & $\begin{array}{l}0 \text { : inexistentes } \\
\text { 2: escasos(menos de un } 10 \% \text { de superficie) } \\
\text { 5: bien representados (igual o mayor al } 10 \% \text { de superficie) }\end{array}$ \\
\hline Especies vegetales amenazadas & $\begin{array}{l}0: \text { inexistentes } \\
\text { 5: representadas }\end{array}$ \\
\hline Especies animales muy amenazadas & $\begin{array}{l}0: \text { inexistentes } \\
\text { 10: representadas }\end{array}$ \\
\hline Especies de aves Directiva Aves Anexo 1 & $\begin{array}{l}0: \text { inexistentes } \\
5: \text { representadas }\end{array}$ \\
\hline Hábitats prioritarios & $\begin{array}{l}0: \text { inexistentes } \\
\text { 10: representados }\end{array}$ \\
\hline Pertenencia a espacios naturales protegidos & $\begin{array}{l}\text { 10: LIC y ZEPA } \\
\text { 5: otros } \\
0: \text { no }\end{array}$ \\
\hline Árboles, comunidades de especies y hábitats excepcionales & $\begin{array}{l}0: \text { no aparecen } \\
\text { 10: aparecen }\end{array}$ \\
\hline Plantas y animales exóticos invasores (puntúa negativamente) & $\begin{array}{l}\text { 0: no aparecen o son de peligrosidad menor } \\
\text { 5: aparecen poblaciones abundantes o de alta peligrosidad } \\
\text { 10: hábitat invadido por completo }\end{array}$ \\
\hline
\end{tabular}


Cuadro 2. Categorías cualitativas del valor de conservación.

Categories of quantifying the value of conservation.

\begin{tabular}{lcl}
\hline \multicolumn{1}{c}{ Calificación } & Puntuación & \multicolumn{1}{c}{ Justificación } \\
\hline Valor óptimo & 95 & $\begin{array}{l}\text { Máximo valor de conservación } \\
\text { Existían al menos once atributos de los catorce positivos y entre ellos siempre los } \\
\text { de mayor peso. } \\
\text { Como mínimo tenían valor en todos los atributos positivos, aunque no siempre fuese } \\
\text { el máximo. }\end{array}$ \\
Estado favorable & $80-95$ & $\begin{array}{l}\text { Por lo menos alcanzaban la mitad de los puntos a repartir } \\
\text { Estado aceptable }\end{array}$ \\
Desfavorable & $48-64$ & $\begin{array}{l}\text { atributos de los catorce bien representados. } \\
\text { Tramos muy deprimidos en los que no había más de uno o dos atributos de los } \\
\text { catorce definidos bien representados, siendo el resto escasos o inexistentes }\end{array}$ \\
\hline Muy desfavorable & $30-47$ &
\end{tabular}

ban huecos susceptibles de albergar especies de fauna (quirópteros, roedores y rapaces nocturnas entre otros), particularmente los realizados por pájaros carpinteros (familia Picidae), que terminan siendo ocupados por los anteriores y árboles que presentan hongos yesqueros (hongos saprófitos de la madera), que dan al tronco unas características de aislamiento frente a la humedad $\mathrm{y}$ al frío muy buscadas por la fauna. Al igual que en caso anterior, se asignó una puntuación de 0 cuando eran inexistentes, de 2 cuando eran escasos (1-5 pies/ha) y 5 cuando eran numerosos ( $>5 \mathrm{pies} / \mathrm{ha}$ ).

6. Presencia de medios abiertos ricos en flores. Estos medios suponen la atracción principal de insectos, no solamente de lepidópteros y de himenópteros como los Bombus spp. en verano, sino de buena parte de invertebrados, ya que estos se acercan durante el estiaje atraídos por la floración de muchas especies que se mantienen por la frescura del terreno en este hábitat (frente al resto de herbazales agostados). También la producción de flores y semillas sirve de alimento a roedores como Apodemus sylvaticus L, una de las presas frecuentes del visón europeo (Mustela lutreola L.) en España, así como a diversas aves rapaces amenazadas. Dentro de estos medios también se consideraron los cardales con numerosas especies de plantas nutricias para larvas de lepidópteros y cuyas flores alimentan a los adultos, así como numerosas especies de invertebrados. Para las riberas del ámbito mediterráneo se consideró adecuada la presencia de un mínimo de un $10 \%$ de sitios abiertos de estas características, por lo que por debajo de ese porcentaje se calificaron de "escasos" y se asignó una puntuación de 2 . Por encima de esa cifra se consideraron "bien representados" recibiendo una puntuación de 5; y cuando estas formaciones no existieron, se consideraron "inexistentes" y la puntuación fue de 0 .

7. Presencia de medios acuáticos: humedales y estanques. Importantes como paraje de cría y alimentación para numerosas especies de invertebrados (cangrejos y diversos insectos) y vertebrados como aves y anfibios (Arvicola sapidus Miller.). Estos últimos a su vez sirven de alimento a especies amenazadas (Lutra lutra L., Mustela lutreola, ardeidas y otras aves acuáticas amenazadas, como Circus aeruginosus L.). Estos humedales tienen un valor estratégico para la conservación de lepidópteros, debido a que usan sus bordes como abrevaderos en verano y épocas de estiaje. Al igual que en el caso anterior, se asignó una puntuación de 2 (menos de un $10 \%$ de superficie ocupada por medios acuáticos), de 5 (más de un $10 \%$ de superficie ocupada por medio acuáticos) o de 0 (no había representación).

8. Presencia de medios pedregosos o gleras fluviales pertenecientes a la alianza fitosociológica Glaucion flavi. Indican una naturalidad del régimen fluvial fluctuante y sirven de notable referencia en cuanto a la naturalidad de la dinámica fluvial, así como a la recarga de acuíferos. $\mathrm{Su}$ presencia aseguraba un nicho potencial para la regeneración de las estirpes autóctonas de $P_{o-}$ pulus nigra L., especialmente de Populus nigra betulifolia (Pursh) W.Wettst, ligado a marcados cambios de caudal en los ríos que dan lugar a avenidas capaces de remover las gleras. Estos lugares permiten así mismo la nidificación de especies de zonas abiertas, como Charadrius dubius Scopoli. o Actitis hypoleucos L., entre otros. Se otorgó una puntuación de 0,2 o 5 puntos en función de si la presencia de este tipo de zonas en la ribera era inexistente, escasa (superficie menor de un $10 \%$ ) o bien representada (superficie igual o mayor del $10 \%$ ), respectivamente.

9. Presencia de especies vegetales amenazadas. Se valoró la existencia de i) clones de procedencias autóctonas de Populus nigra nigra L., Populus nigra betulifolia y Populus nigra caudina (Ten.) Bugała, abundantes en zonas del norte de la Península Ibérica pero que a nivel europeo se encuentran en grave peligro de extinción (Koskela et al. 2004), ii) ejemplares de Ruscus aculea- 
tus L. presente en la Directiva Hábitats (92/43/CEE), y iii) poblaciones autóctonas de nogal asimilables a Juglans regia L. subsp. hispanica D. Rivera et al., considerada autóctona y de interés especial (Carrión y Sánchez-Gómez, 1992). En el caso de existir alguna de las especies indicadas la puntuación sería de 5 puntos y en caso contrario de 0 puntos.

10. Presencia de especies animales muy amenazadas según la Directiva Hábitats. Se valoró la existencia (5 puntos) o no (0 puntos).

11. Presencia de especies de aves del Anexo 1 de la Directiva Aves (79/409/CEE), cuya conservación es necesaria y cuya presencia es habitual en el ámbito de estudio. Se valoró la existencia (5 puntos) o no (0 puntos).

12. Presencia de hábitats prioritarios. Se valoró la existencia (5 puntos) o no (0 puntos) de hábitats de obligada conservación que aparecen codificados en la Directiva Hábitats (Escudero et al. 2008).

13. Pertenencia a espacios naturales protegidos. Se valoró la existencia de zonas sujetas a figuras de protección de diversa índole como pueden ser Lugares de Interés Comunitario (LIC) y Zonas de Especial Protección para las Aves (ZEPA), que son áreas catalogadas por los estados miembros de la Unión Europea (10 puntos) u otras (5 puntos).

14. Presencia de árboles, comunidades de especies y hábitats excepcionales. Se valoró (10 puntos) aspectos de orden excepcional, como eran grandes árboles monumentales (ejemplares de Quercus ilex L. gigantescos, entre otros), rodales muy escasos y amenazados como las olmedas de Ulmus minor Mill (extinguidos en muchas zonas por la grafiosis del olmo) y/o estirpes escasas como el caso de Quercus robur broteroana Schwarz y Ulmus laevis Pall. Además, se incluyeron destacados nocedales, es decir, rodales de nogal asimilables a Juglans hispanica, así como aquellos parajes que albergaban colonias de numerosos nidos de aves catalogadas y aquellas áreas de alto valor de diversidad, como los tramos donde el número de especies de lepidópteros superaban las 25 especies.

15. Presencia de especies vegetales y animales exóticas invasoras. Se valoró con puntuación negativa la mayor o menor presencia de especies invasoras agresivas, como podían ser Ailanthus altissima (Miller) Swingle, Cortaderia selloana (Schult et Schult F.) Asch et Graebn, Arundo donax L. y Salix babylonica L. entre las especies vegetales y Mustela vison, Schreber como especie animal; o de menor peligrosidad como Robinia pseudacacia L., Populus x euramericana Dode y Populus $x$ interamericana Brokehuizen. De este modo se puntuó con -10 puntos cuando el hábitat aparecía invadido por completo por alguna de estas especies, con -5 puntos cuando aparecían especies invasoras peligrosas o de forma abundante las especies menos peligrosas y 0 puntos cuando no aparecían especies peligrosas o lo hacían de forma escasa las de menor peligrosidad.
El caso de los clones de interés en populicultura resultó peculiar, ya que se trata de árboles que alcanzan fácilmente alturas y diámetros considerablemente mayores que $\mathrm{Po}_{\mathrm{O}}$ pulus nigra debido a su capacidad de crecimiento, siendo muy atractivos para numerosas especies de animales, sobre todo de aves que los utilizan para nidificar. En algunas provincias españolas las amenazadas águilas imperiales ibéricas anidan en estas estirpes híbridas antes que en otras especies de árboles de ribera autóctonas de menor porte. Debido a lo expuesto anteriormente estos clones en caso de no existir árboles autóctonos de gran tamaño, no se consideraron negativas.

Esta nueva metodología también permitió identificar zonas susceptibles de ser clasificadas como MAVC y valorar el estado de conservación de las mismas. El concepto de MAVC fue creado por FSC y se define como la Unidad de Gestión Forestal (UGF) o parte de ella, que contiene uno o más de los seis atributos que se indican en documentos del propio Consejo (García et al. 2010) y que se incluyen dentro de los 15 atributos definidos en el método propuesto. Por tanto, se puede hablar de seis tipos de MAVC en función de qué atributo contenga la zona analizada. La identificación de estos MAVC es muy importante ya que los estándares de certificación forestal FSC contienen unos requisitos específicos para estos Montes (García et al. 2010), por lo que para conseguir la certificación FSC, deben estar previamente identificados y proponer una gestión forestal específica para ellos que asegure su mantenimiento o mejora. La metodología anterior (cuadro 1 y 2) permite identificar los MAVC del tipo 1 hasta el 5, de tal forma que aquellas UGF que tengan valor positivo en uno o varios de los atributos o características decisorias 3, 10, 11, 12, 13 y/o 14 del método propuesto (atributos comunes con los definidos por FSC), serían consideradas como MAVC.

El caso concreto de los MAVC tipo 6 (o que contengan el atributo 6 de los indicados por FSC), se analiza por separado. Se trata de UGF que presentan un valor específico para Comunidades locales y que son seleccionados como MAVC por "ser fundamentales para la satisfacción de las necesidades básicas de las comunidades locales (e.g. subsistencia, salud) y/o críticas para su identidad cultural tradicional (áreas de importancia cultural, ecológica, económica o religiosa, identificadas en cooperación con dichas comunidades locales)" (García et al. 2010). En este caso no se puede ni se debe aplicar la valoración y evaluación del mismo modo que en los otros tipos de MAVC, ya que el valor del ecosistema está ligado a valores estéticos, espirituales, educacionales y recreativos, que no aparecen entre los atributos reflejados en el cuadro 1. Por tanto, la nueva metodología, contempla una segunda lista de atributos para identificar el estado de conservación de estos MAVC tipo 6 (cuadro 3), con sus ponderaciones correspondientes, entre los que se incluyeron esos valores especiales o beneficios ecosistémicos (HCV Network 2013).

En la zona de estudio, los valores especiales venían dados fundamentalmente por la presencia de dos estructuras 
Cuadro 3. Ficha de ponderación de variables cualitativas para MAVC tipo 6.

Data sheet weighting of qualitative variables of MAVC type 6.

\begin{tabular}{|c|c|c|}
\hline Atributo & Descripción de los elementos a valorar & Criterio de valoración \\
\hline Valor estético del paisaje & $\begin{array}{l}\text { Estéticamente el tramo a valorar tenía las mismas características } \\
\text { que el espacio con valores especiales en ese lugar. }\end{array}$ & $\begin{array}{l}\text { 0: no representativo } \\
\text { 2: baja representatividad } \\
\text { 5: representatividad media } \\
\text { 10: alta representatividad }\end{array}$ \\
\hline $\begin{array}{l}\text { Capacidad de acogida de } \\
\text { visitantes }\end{array}$ & $\begin{array}{l}\text { Estas zonas son visitadas por gran cantidad de personas. } \\
\text { Aquí se valoró la proporción de superficie analizada que está } \\
\text { preparada para recibirlos, (mobiliario y equipamiento). }\end{array}$ & $\begin{array}{l}0: \text { ninguna parte } \\
\text { 2: una pequeña parte } \\
\text { 5: escasa } \\
\text { 10: media } \\
\text { 20: la mayor parte }\end{array}$ \\
\hline Transitabilidad & $\begin{array}{l}\text { Estaba adaptado al tránsito de las personas, evitando que la } \\
\text { vegetación natural se desarrollase tanto que impidiese el paso. }\end{array}$ & $\begin{array}{l}\text { 0: intransitable } \\
\text { 2: poco transitable } \\
\text { 5: transitable con alguna dificultad } \\
\text { 10: transitable }\end{array}$ \\
\hline Limpieza & Presencia de basura y escombros & $\begin{array}{l}\text { 0: alta acumulación de residuos } \\
\text { 5: baja acumulación de residuos } \\
\text { 10: sin residuos }\end{array}$ \\
\hline Valor divulgativo & $\begin{array}{l}\text { Existe información en forma de carteles donde se explique el } \\
\text { interés del paisaje, la fauna y la flora del entorno a los usuarios } \\
\text { de la zona. }\end{array}$ & $\begin{array}{l}0: \text { no } \\
10: \text { sí }\end{array}$ \\
\hline Valor educativo & $\begin{array}{l}\text { Presencia de valores naturales reseñables, como podían ser } \\
\text { los procesos geomorfológicos o la presencia de paisajes } \\
\text { determinados entre otros. }\end{array}$ & $\begin{array}{l}0: \text { no } \\
\text { 10: sí }\end{array}$ \\
\hline Valores naturales del espacio & Flora, fauna y hongos característicos & $\begin{array}{l}\text { 0: no aparecen } \\
\text { 2: escaso valor } \\
\text { 5: valor medio } \\
\text { 10: alto valor }\end{array}$ \\
\hline $\begin{array}{l}\text { Valor como refugio de } \\
\text { transeúntes }\end{array}$ & Árboles con capacidad de dar sombra & $\begin{array}{l}\text { 0: sin árboles } \\
\text { 5: escasos (1 a } 10 \text { árboles/ha) } \\
\text { 10: frecuentes ( }>10 \text { árboles/ha) }\end{array}$ \\
\hline
\end{tabular}

lineales emblemáticas del norte peninsular como son el Camino de Santiago y el Canal de Castilla. Los atributos se particularizaron para estos ejemplos pero análogamente pueden particularizarse para otros casos de aplicación (cuadro 3). La suma de estos atributos daba lugar al valor de conservación de los MAVC tipo 6. Dicho valor de conservación se interpretó conforme a lo dispuesto en el cuadro 4.

Los atributos se definieron, en algunos casos, atendiendo a figuras legales o normativa europea. En caso de aplicar este método fuera de Europa habría que ajustarlo atendiendo a las figuras legales y a la legislación que sea de aplicación en estos lugares.

Aplicación de la nueva metodología en casos de estudio. Como ejemplo de uso de la metodología propuesta se planteó su aplicación en diversas parcelas pertenecientes a los cauces de algunos de los principales ríos de la submeseta norte española. De este modo, inicialmente se analizaron 33 parcelas localizadas en las cuencas hidrográficas de los ríos Duero y Sil (León) (figura 1A), y 16 en la cuenca del río Ebro (La Rioja) (figura 1B). Estas parcelas estaban gestionadas por una empresa maderera que necesitaba valorar su estado de conservación para mantener el certificado FSC de las mismas. Posteriormente, para validar la metodología en otros lugares, se aplicó a dos cauces completos (ríos Carrión y Valdavia), con características muy diferentes entre ellos: 1) el río Carrión, que discurre por la provincia de Palencia, con un desnivel de más de 800 metros, una longitud principal de 178,5 kilómetros y drena una superficie de 3.351 kilómetros cuadrados; y 2) el río Valdavia, que transita por las provincias de Burgos y Palencia, tiene únicamente 71 kilómetros de longitud y presenta un acusado estiaje en verano. En este último estudio, la longitud de cada cauce se dividió en una serie de tramos de tal forma que se analizaron 41 tramos del río Carrión y nueve del río Valdavia (figura 1C). Para el establecimiento de los tramos de estudio en cada cauce, inicialmente se utilizó cartografía y ortofotos referidas a una fecha concreta (2.005). A base de esta cartografía e imágenes se delimitaron los 
diferentes tramos, entendiéndose por tramo a efectos de este trabajo como la longitud del cauce cuya vegetación de ribera estuviera formada por una mancha homogénea de vegetación distinguible de las de su entorno y delimitadas, siempre que fuera posible, por líneas naturales como ríos y arroyos, entre otros, o artificiales, como carreteras, caminos o cortafuegos, bien definidas e identificables. Esta delimitación facilitaba la localización del tramo sobre el terreno para su primera valoración y posible seguimiento posterior. Por tanto, cada tramo tuvo una longitud y superficie diferente.

Una vez se delimitaron las parcelas y los tramos, se recorrieron la totalidad de las superficies en verano y comienzos de otoño, entre los meses de junio y octubre (época de mayor actividad en estos ecosistemas en el Hemisferio Norte). En los recorridos se recogieron todos

Cuadro 4. Categorías cualitativas del valor de conservación para zonas con valores especiales: estéticos, espirituales, educacionales y/o recreativos.

Categories of quantifying the value of conservation for areas with special values: aesthetic, spiritual, educational and/or recreational.

\begin{tabular}{lcl}
\hline \multicolumn{1}{c}{ Calificación } & Puntuación & \multicolumn{1}{c}{ Justificación } \\
\hline Valor óptimo & 90 & $\begin{array}{l}\text { Máximo valor de conservación. } \\
\text { Presentaba todos los atributos definidos, aunque no siempre la valoración del } \\
\text { atributo era máxima. }\end{array}$ \\
Estado muy favorable & $80-90$ & $\begin{array}{l}\text { Presentaba al menos el } 75 \% \text { de los atributos definidos, aunque no siempre la } \\
\text { valoración del atributo era máxima. }\end{array}$ \\
Estado favorable & $65-79$ & $\begin{array}{l}\text { Presentaba al menos el } 60 \% \text { de los atributos definidos, aunque no siempre la } \\
\text { valoración del atributo era máxima. }\end{array}$ \\
Desfavorable & $48-64$ & $\begin{array}{l}\text { Presentaba al menos el 25\% de los atributos definidos, no alcanzando el } 60 \% . \\
\text { No llegaba al } 25 \% \text { de los atributos definidos con una buena representación. }\end{array}$ \\
Muy desfavorable & $30-47$ & $<30$
\end{tabular}

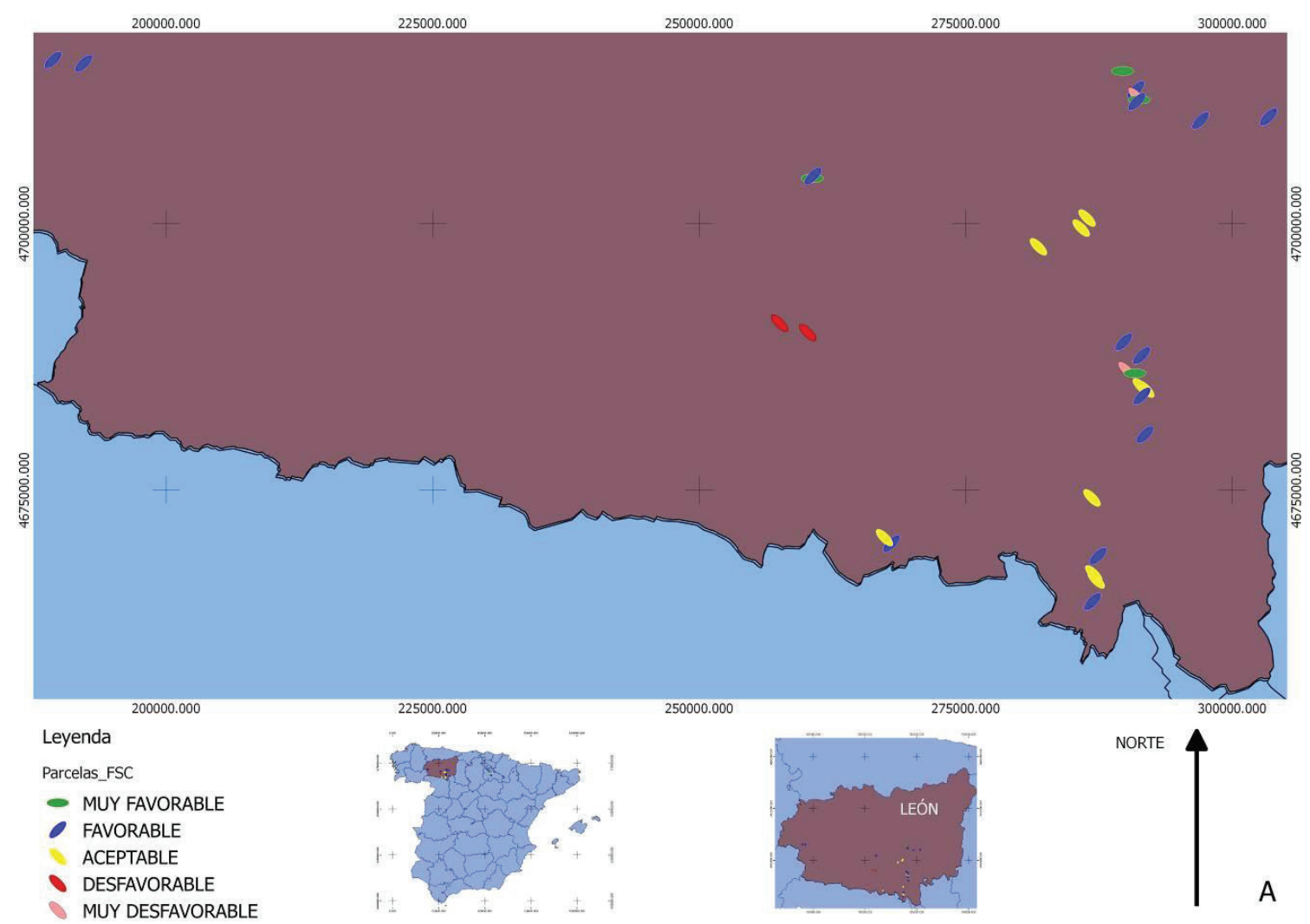

Continúa 

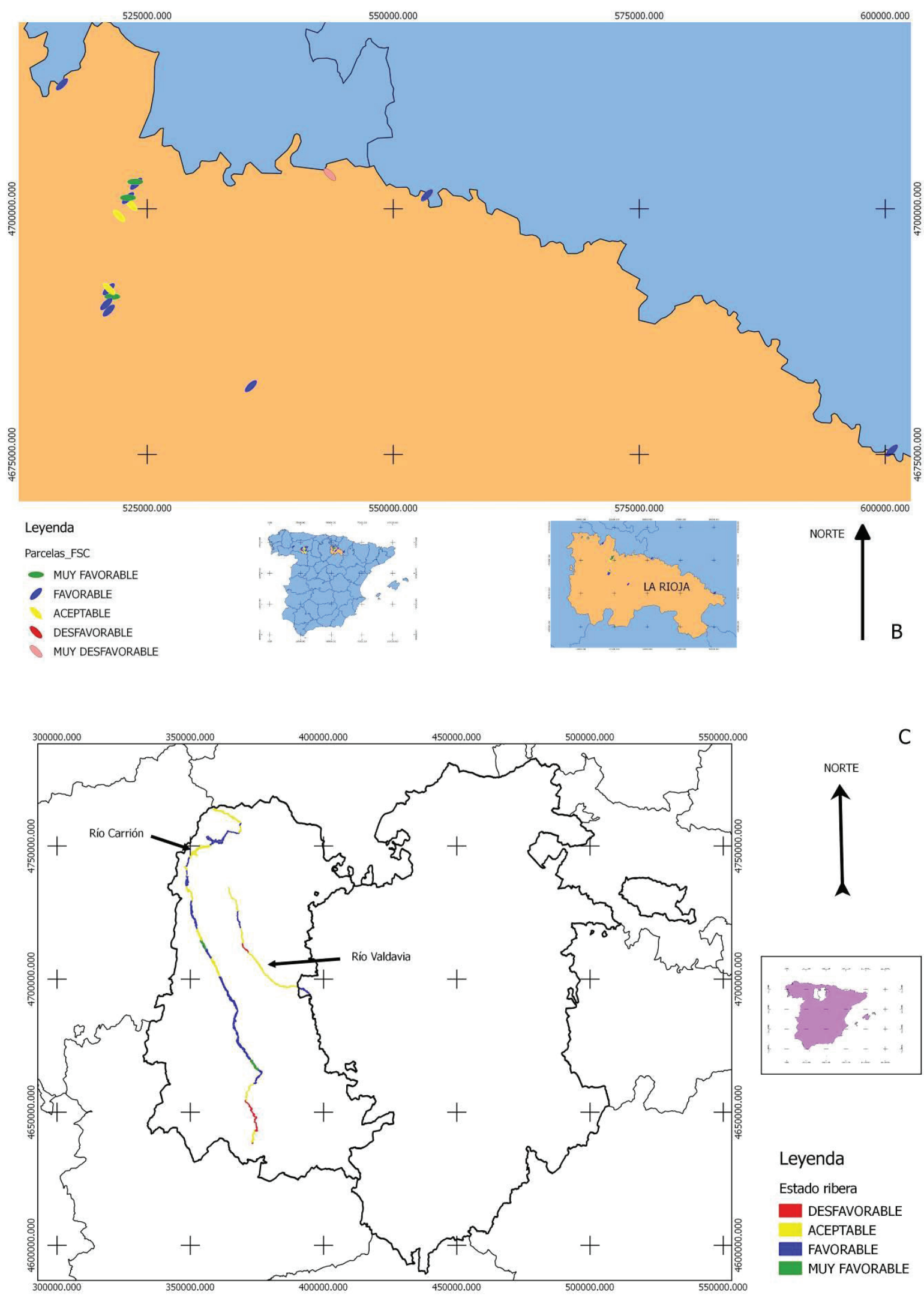

C

ORT
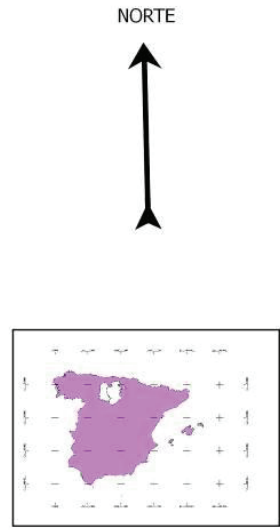

Leyenda

Estado ribera

DESFAVORABLE

ACEPTABLE

FAVORABLE

MUY FAVORABLE

Figura 1. Mapas de calidad de las parcelas de León (A) y La Rioja (B) y las riberas de los cauces del Carrión y del Valdavia (C) aplicando el método referido.

Quality map of the plots of León (A) and La Rioja (B) and the banks of Carrión and Valdavia channels (C) applying the referred method. 
los datos de cada parcela o tramo: florísticos, faunísticos, de usos tradicionales, vegetación climatófila adyacente, anchura de la ribera, actividades que se realizaban en el mismo y los impactos que se producían en estos lugares. Posteriormente, en gabinete se completó esta información con los datos relativos a su localización, descripción de morfometría, clima, geología y fauna a partir de bibliografía. Con los datos obtenidos se determinó su estado de conservación utilizando la metodología descrita en el anterior apartado, identificándose los tramos que podían ser considerados MAVC según lo indicado.

\section{RESULTADOS}

Las 33 parcelas realizadas en la provincia de León mostraron de modo mayoritario (84,85\% de las parcelas) un estado de conservación que podía caracterizarse como positivo o muy positivo. Se encontraron varias parcelas en situación desfavorable $(9,09 \%$ del total) e incluso dos parcelas en situación muy desfavorable (figura 2A). Estas últimas no llegaban a ser MAVC (6,06\% de las parcelas), por no poseer atributo alguno de los definidos por FSC.

En numerosas ocasiones apareció el hábitat prioritario formado por alisedas occidentales en mezcla con otras especies (bosque aluvial código 91EO de la Directiva Hábitats de la Unión Europea) y el hábitat prioritario TheroBrachypodietea (Código 6220* de la Directiva Hábitats).

A
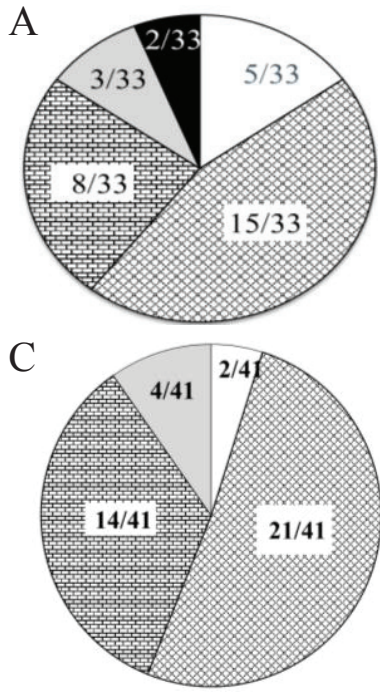

$\square$ MUY FAVORABLE

Q FAVORABLE

- MUY DESFAVORABLE

Figura 2. Resultados globales del estado de conservación de los tramos de León (A), de La Rioja (B), del río Carrión (C) y río Valdavia (D) según número de tramos analizados.

Overall results of the state of conservation of the sections of León (A), La Rioja (B), Carrión River (C) and Valdavia River (D) according to the number of sections.
Cabe destacar que por cuatro de las parcelas estudiadas transcurría el Camino de Santiago. Estos lugares unieron a su interés natural por incluir un hábitat prioritario, unas características especiales (función de índole social, estética, espiritual y recreativa), que acrecentó de modo particular su valía. En estas zonas, destacó así mismo la presencia casi constante de animales de gran valor como la nutria (Lutra lutra), que no solamente ocupaban los terrenos inmediatos al cauce de los ríos sino también las áreas más alejadas.

En las parcelas de estudio de La Rioja el estado de conservación de acuerdo con la metodología de evaluación resultó en su mayoría favorable (figura $2 \mathrm{~B}$ ). El número de parcelas que alcanzó un nivel muy favorable fue del 18,75\%. El $62,5 \%$ del total obtuvo un nivel favorable, no detectándose parcelas en estado desfavorable y únicamente una muy desfavorable, ver situación en la figura $1 \mathrm{~B}$, no presentando atributo alguno de los definidos por FSC para ser MAVC.

De igual forma que en el caso anterior, volvían a aparecer con frecuencia el bosque aluvial de Alnus glutinosa junto con avellanedas ribereñas de Corylus avellana L. silvestre (código 91EO de la Directiva Hábitats de la Unión Europea) y el hábitat prioritario Thero-Brachypodietea (Código 6220* de la Directiva Hábitats). Es importante destacar la presencia del visón europeo en casi todas las parcelas analizadas en La Rioja y de abundante presencia de zarzales-espinares diferenciados en las riberas, requerimiento de esta especie de visón para su conservación. Paralelamente, una de las peores amenazas que se constató fue la presencia constante del visón americano, máximo competidor directo para el visón europeo y que además, es un fiero eliminador de otras especies de fauna silvestre amenazada.

En el río Carrión los resultados (figura 2C) mostraron como de modo mayoritario (90,24\%), las parcelas constataron un estado de conservación que podía caracterizarse como positivo o muy positivo. Varias parcelas mostraron estar en situación desfavorable $(4,88 \%$ del total).

Aparecieron en numerosas ocasiones hábitats prioritarios, como turberas acidófilas (Turberas altas activas, Código 7110* de la Directiva Hábitats), zonas subestépicas de gramíneas y anuales del Thero-Brachypodietea (Código 6220* de la Directiva Hábitats), formaciones herbosas con Nardus, con numerosas especies, sobre sustratos silíceos de zonas montañosas (Código 6230* de la Directiva Hábitats) y bosques aluviales de Alnus glutinosa y Fraxinus excelsior L. (Alno-Padion, Alnion incanae, Salicion albae, Código 91E0* de la Directiva Hábitats), lo que confería a la zona de estudio un elevado interés natural. Por otro lado, se comprobó que el hecho de que tanto el Camino de Santiago (a su paso por Carrión de los Condes), como el Canal de Castilla (a su paso por Calahorra) cruzasen el río Carrión, incrementaban su valía en el ámbito sociocultural y espiritual.

En el río Valdavia los resultados (figura 2D) mostraron cómo de modo mayoritario $(86,89 \%)$ el estado de conservación podía caracterizarse como positivo, aunque la 
mayor parte de las parcelas $(66,67 \%)$ mostraron un estado de conservación aceptable. Se identificó, no obstante, una parcela en situación desfavorable (11,11\% del total). En la figura $1 \mathrm{C}$ se observa el grado de calidad de cada tramo.

Aun así, en los tramos analizados apareció en numerosas ocasiones el hábitat prioritario Zonas subestépicas de gramíneas y anuales del Thero-Brachypodietea (Código 6220* de la Directiva Hábitats). Destacó además la presencia casi constante de especies animales de gran valor como la nutria. En lo referente al valor sociocultural, el tramo más relevante resultó ser el del cruce con el Canal de Castilla, lugar en el que se localiza el espectacular puenteacueducto de Abánades, con sus cinco arcadas que permite al Canal de Castilla salvar las aguas del río Valdavia.

Si comparamos los resultados obtenidos para los dos cauces completos analizados, comprobamos la existencia en el río Carrión de una mayor diversidad de ecosistemas y un mayor número de espacios protegidos, animales y especies vegetales en peligro de extinción y hábitats prioritarios que en el río Valdavia. Aun así, en ambos casos todos los tramos estudiados presentaban alguno de los atributos definidos por FSC para ser declarados MAVC.

\section{DISCUSIÓN}

Metodología propuesta. La gran facilidad de aplicación de la metodología propuesta la convierte en una herramienta valiosa para la gestión, ya que permite convertir la información recogida en campo (presencia de especies vegetales, animales y/o madera muerta entre otras), en valores numéricos con los que cuantificar de forma más objetiva el estado de conservación de una zona. Es importante destacar que uno de los puntos fuertes de la nueva metodología propuesta en este trabajo para la evaluación del estado de conservación de riberas es que puede ser aceptada en los procesos de certificación ambiental. Esto no ocurre con el resto de metodologías por no incluir los atributos que se valoran en dichos procesos. De hecho el análisis de las parcelas de León y La Rioja, ya ha sido aceptado por FSC para localizar lugares que cumplen con la normativa impuesta por FSC para ser MAVC. Así mismo, este método cobra especial valor porque, además de los valores que ya se recogen en metodologías anteriores, por primera vez se tienen en cuenta novedosos valores como i) los aspectos legales de conservación; ii)la identidad cultural, social y/o espiritual presente en la zona; iii) la problemática ambiental; y iv)la biodiversidad de paisajes para la conservación que son catalogados como importantes tanto en la Directiva Hábitats Europea (Escudero et al. 2008), como en la valoración ambiental en la certificación (Dufour y Piégay 2006, Emberger et al. 2015, Abell et al. 2015).

Son muchos los países como Chile, que tienen incentivos para la restauración y protección del bosque en las zonas ribereñas que incluyen bonificaciones, exención de impuestos o pago por servicios ambientales en aquellas zonas que están certificadas (Kosoy et al. 2007). Además, se considera fundamental el desarrollo de estudios científicos que caractericen la vegetación, la dinámica de la regeneración y la propagación de plantas en las zonas ribereñas para la correcta implementación de actividades de recuperación y restauración de estos ecosistemas de características singulares en cuanto a la distribución espacio-temporal de sus componentes biológicos (Romero et al. 2014). La existencia de un método como el propuesto, que es aceptado en los procesos de certificación ambiental, constituye un impulso tanto para las grandes empresas que buscan obtener la certificación y una contribución a una demanda de la sociedad en la actualidad, como para los pequeños propietarios de bosques en las zonas rurales.

Petersen (1992) fue pionero en este sentido con la propuesta de un método que valora dieciséis atributos también muy variados. Sin embargo, estos son difíciles de aplicar y deja lugar a la subjetividad por lo que resulta complicada la comparación de estudios realizados por diferentes personas con este método. Por otra parte, el método REC (Snelder y Biggs 2002) se basa únicamente en aspectos climatológicos, morfológicos y geológicos, por lo que no se puede utilizar para valorar el estado de la vegetación de ribera. Posteriormente este método fue adaptado por Peredo-Parada et al. (2011) denominándolo REC-Chile, incluyendo un nuevo aspecto con vistas a la conservación de los ecosistemas acuáticos, no a los terrestres. Los métodos QBR e IHF han demostrado ser de gran utilidad y efectividad a la hora de evaluar de manera rápida y objetiva la calidad de los ríos de Chile (Palma et al. 2009), pero no detectan todos los atributos que requiere FSC para la certificación forestal. Tanto en estas metodologías como en el RQI (González del Tánago et al. 2006), se desprecian e ignoran, e incluso se valoran negativamente las zonas sin bosques altos y complejos, a pesar de que pueden ser objeto de certificación si cumplen una serie de atributos como los que se recogen en el método aquí expuesto. Además la Directiva Hábitats de la Unión Europea da un gran apoyo a la conservación de este tipo de hábitats abiertos y esteparios, proporcionando un valor PRIORITARIO a los numerosos pastizales secos de Thero Brachypodietalia (Escudero et al. 2008), localizados en el ámbito mediterráneo y muy presentes en las riberas de los ríos españoles.

Con el método propuesto se da importancia también a comunidades como los zarzales-espinares, detectados como necesarios para la fauna amenazada. En otros métodos de valoración se consideran etapas de degradación del bosque, pese a que suponen una protección de los ecosistemas, que ayudan a que la regeneración de árboles de los géneros ribereños se vea protegida por esta orla espinosa de arbustos. Además suponen un extraordinario valor para la fauna, siendo sus flores una gran atracción para numerosos insectos polinizadores de todo tipo (himenópteros y lepidópteros, entre otros).

Estado de conservación de los cauces. Las parcelas de las cuencas del Sil, Duero y Ebro fueron las que presentaron 
mayores porcentajes de parcelas muy bien conservadas (figura $2 \mathrm{~A}$ y B). Esto se relaciona tanto con su localización en lugares con escasa población humana (provincia de León), lo que provoca una menor presión sobre la vegetación, como con el hecho de que en estas zonas los cultivos de regadío intensivos (maíz y remolacha fundamentalmente) han sido sustituidos en las últimas décadas por choperas productivas, reduciéndose enormemente el uso de productos químicos fitosanitarios, lo que ha tenido un efecto positivo sobre la vegetación de ribera. Este efecto positivo se ha podido comprobar por la existencia de bosques naturales en las zonas de estudio que tienen una edad de 15-20 años en el caso de las parcelas de León y de 30 a 50 años en La Rioja.

Con respecto a los ríos analizados en las provincias de Palencia y Burgos, el estado de conservación del río Carrión es superior al río Valdavia (figura $2 \mathrm{C}$ y D). Esto coincide con la existencia en el río Carrión de una mayor diversidad de ecosistemas, un mayor número de espacios protegidos, animales y especies vegetales en peligro de extinción, así como hábitats prioritarios, localizándose los valores menores en zonas cercanas a grandes poblaciones donde la alteración y degradación de las riberas es mayor.

Recomendaciones de gestión. Esta metodología se muestra útil en el Hemisferio septentrional y aplicable en el Hemisferio meridional donde se considera muy interesante realizar un estudio que compare este método junto con el REC-Chile, el QBR y el IHF. Estas son metodologías que se utilizan con éxito en los ríos de Chile de forma que si la correlación entre los datos obtenidos con los cuatro métodos es similar, determinará la adaptación de este método a este país y su posible uso para la certificación de bosques nativos en las zonas rurales pertenecientes a comunidades y pequeños propietarios. Además, se cree oportuno comprobar que puede ser utilizado en ecosistemas alejados de las riberas, adaptándolo en cada caso a la idiosincrasia del lugar, de manera que puedan ser comparados diferentes ecosistemas valorados con este método, algo que no puede hacerse con los métodos existentes hasta ahora por la especialización de los mismos.

Atendiendo a los resultados obtenidos y dependiendo de si se quiere mantener o mejorar el estado de conservación las actuaciones a realizar serán diferentes. En el caso de que se quiera mantener el nivel actual, deben realizarse labores que mantengan el estado de las comunidades allí presentes. Esto puede conseguirse con actuaciones relativamente sencillas como puede ser entre otras:

- Una presencia ganadera que favorezca la conservación de hábitats como el prioritario Thero-Brachypodietalia o el no prioritario de las junqueras mediterráneas, $o$

- Con la realización de desbroces, podas y desmoches que mantengan una masa saneada y puedan incluso incrementar el número de oquedades y re- fugios y aporte de madera muerta en la zona, entre otras actuaciones.

Si lo que se quiere es mejorar el estado de conservación de los tramos por considerarlos prioritarios, se propone:

- Fomentar la biodiversidad en cuanto a especies arbóreas y subarbóreas mediante siembras y plantaciones. El resultado de una mayor biodiversidad vegetal supondrá una mayor variedad de alimento así como de refugios que con el tiempo favorecerán la instalación de nuevas especies de fauna.

- Evitar la entrada de especies exóticas tanto animales como vegetales. Para ello se vigilarán las zonas y se eliminaran las especies invasoras en el momento en el que se detecte el primer ejemplar. Estas especies se ha visto como desplazan a las especies autóctonas, empobreciendo la biodiversidad de la zona.

Existen otras actuaciones positivas, aunque se cree algo menos efectivas para la mejora del estado de conservación por afectar a un menor número de atributos, como son:

- Promover la formación de charcas con superficie de agua libre valiosa para la diversidad de animales.

- Colocar cajas de cría para la fauna.

Además, se considera interesante ampliar la superficie de ribera con la plantación en la banda más externa respecto al cauce del río, con especies como Fraxinus angustifolia, Fraxinus excelsior, Juglans regia y Quercus pyrenai$c a$, que ofrecen un alimento (forraje y frutos) que puede ser utilizado por el ganado y por la fauna salvaje existente en estos parajes.

El seguimiento anual y quinquenal con este método de un mismo tramo permite determinar si la gestión que se está llevando a cabo en la zona es beneficiosa o perjudicial para la misma. Asimismo, se considera muy interesante aplicar este método antes y después de una recuperación y/o restauración hidrográfica, ya que indica la eficacia de la misma.

Por último, cabe destacar que es interesante promover un turismo que cada día valora más este tipo de espacios. Además de conservar los valores culturales existentes en algunas de estas riberas, como es el Camino de Santiago y el Canal de Castilla, respaldando y alentando actividades de investigación, de educación, de formación y de observación permanentes encaminadas a la conservación y el desarrollo sostenible.

\section{CONCLUSIONES}

La metodología propuesta en este trabajo supone una valiosa herramienta para caracterizar el estado de conservación de las riberas, valorar la efectividad de la gestión que se esté realizando y poder conseguir la certificación forestal FSC. 


\section{REFERENCIAS}

Abell R, SK Morgan, AJ Morgan. 2015. Taking High Conservation Value from Forest to Freshwaters. Environmental Management 56:1-10.

Bjorkland R, CM Pringle, B Newton. 2001. A stream visual assessment protocol (SVAP) for riparian landowners. Environmental Monitoring and Assessment 68 (2): 99-125.

Campodron J. 2008. Elementos biológicos a tener en Cuenta en la planificación forestal a escala de rodal y de paisaje. Cuadernos de la Sociedad Española de Cienciass Forestales 27: 79-86.

Carrión JS, P Sánchez-Gómez. 1992. Palynological data in support of the survival of walnut (Juglans regia L.) in the western Mediterranean area during last glacial times. Journal of Biogeography 19: 623-630.

Dufour S, H Piégay. 2006. Forêts riveraines et ripisylves: spécificités, fonctions et gestion. Revue Forestière Française 4: 339-350.

Emberger C, P Gonin, L Larrieu. 2015. L'Indice de Biodiversité Potentielle (IBP): quelles perceptions et utilisations en forêt privée? Résultats d'une enquête menée auprès de propriétaires forestiers. Foret-Entreprise 224: 58-63.

Escudero Alcántara A, JM Olano Mendoza, R García Camacho, P Bariego Hernandez, C Molina Martín, JA Arranz Sanz, JI Molina García, FJ Ezquerra Boticario. 2008. Guía básica para la interpretación de los hábitats de interés comunitario en Castilla y León. Valladolid, España. Junta de Castilla y León, Consejería de Medio Ambiente. 432 p.

García FJ, R Tapias, M Fernández, FJ Vázquez, L Salvador. 2010. La biodiversidad como herramienta en la gestión y certificación forestal: zonas de alto valor de conservación en montes madereros del suroeste peninsular. Boletín informativo CIDEU 8(9):57-73.

González del Tánago M, D García de Jalón Lastra, F Lara, R Garilleti 2006. Índice RQI para la valoración de las riberas fluviales en el contexto de la Directiva Marco del Agua. Ingeniería Civil 143: 97-108.

HCV Resource Network. 2013. Common Guidance for the Identification of High Conservation Values. Consultado ene. 2015. Disponible en https://www.hcvnetwork.org/

Jansen A, A Robertson, L Thomson, A Wilson. 2004. Development and application of a method for the rapid appraisal of riparian condition. River and Riparian Land Management Technical
Guideline 4. Australia, Camberra. Land \& Water. 14 p.

Koskela J, SMG de Vries, D Kajba, G von Wühlisch, compilers. 2004. Populus nigra Network, Report of seventh (25-27 October 2001, Osijek, Croatia) and eighth meetings (22-24 May 2003, Treppeln, Germany). Rome, Italy. International Plant Genetic Resources Institute. $131 \mathrm{p}$.

Kosoy N, M Martínez-Tuna, R Muradian, J Martínez-Alier 2007. Payments for environmental services in watersheds: Insights from a comparative study of three cases in Central America. Ecological Economics (61): 446-455.

Munné A, C Solá, N Prat. 1998. QBR: Un índice rápido para la evaluación de la calidad de los ecosistemas de ribera. Tecnología del Agua 175: 20-37.

Munné A, N Prat, C Solá, N Bonada, M Rieradevall 2003. A simple field method for assessing the ecological quality of riparian habitat in rivers and streams: QBR index. Aquatic Conservation: Marine and Freshwater Ecosystems 13:143-162.

Palma A, R Figueroa, VH Ruiz 2009. Evaluación de ribera y hábitat fluvial a través de los índices QBR e IHF. Gayana 73(1): 57-63.

Pardo I, M Alvarez, J Casas, J Moreno, S Vicas, N Bonada, J Alba-Tercedor, P Jáimez-Cuellar, G Moya, N Prat, S Robles, M Suarez, M Toro, M Vidal-Abarca. 2002. El hábitat de los ríos mediterráneos. Diseño de un índice de diversidad de habitat. Limnetica 21: 115-132

Peredo-Parada M, F Martínez-Capel, DI Quevedo, AB Hernández-Mascarell 2011. Implementation of an eco-hydrological classification in Chilean rivers. Gayana 75(1): 26-38.

Petersen R 1992. The RCE: A riparian, channel and Environmental Inventory for small streams in the agricultural landscape. Freshwater Biology 27: 295-306.

Romero FI, MA Cozano, RA Gangas, PI Naulin. 2014. Zonas ribereñas: protección, restauración y contexto legal en Chile. Bosque 35(1): 3-12.

Snelder TH, BJF Biggs 2002. Multiscale river environment classification for water resource management. Journal of the American Water Resources Association 37: 1-15.

Ward TA, KW Tate, ER Atwill 2003. Visual assessment of riparian health. Rangeland Monitoring Series, ANR Publication 8089. Oakland, California. Regents of the University of California, Division of Agriculture and Natural Resources. 23 p.

Winward AH. 2000. Monitoring the Vegetation Resources in Riparian Areas. Ogden, Utah, USA. USDA Forest Service, General Technical Report RMRS-GTR-47. 49 p. 